\title{
Landeshochschulverbünde in der digitalen Hochschulbildung. Ziele, Leitideen, Synergiepotenziale
}

\section{Tina Ladwig und Christiane Arndt}

\section{Zusammenfassung}

Der vorliegende Beitrag gibt Einblick in das Forschungsprojekt BRIDGING. Im Fokus stehen formalisierte Hochschulverbünde auf Bundeslandebene im Themenfeld Digitalisierung in Studium und Lehre. Ausgehend von einem kurzen (historischen) Überblick über aktuelle Landesinitiativen in Deutschland, werden Motive, Ziele und Leitideen vorgestellt. Danach werden Einschätzungen von Akteur*innen zu möglichen Synergien hinsichtlich einer Bundeslandund damit netzwerkübergreifenden Zusammenarbeit dargelegt. Ziel ist es, eine empirische Annäherung an einen ganzheitlichen bundesweiten Überblick über Motive, Leitideen und Umsetzungsansätze im Rahmen bestehender Netzwerke und Landesinitiativen zu geben. Die Basis hierfür bilden sowohl Expert*inneninterviews als auch eine nicht repräsentative Umfrage in den unterschiedlichen Netzwerken.

\section{Schlüsselwörter}

Kooperationen $\bullet$ Hochschulverbünde $\bullet$ Landesinitiativen $•$ Motive $•$ Ziele $•$ Leitideen

T. Ladwig (凶)

NIT Northern Institute of Technology Management, Hamburg, Deutschland

E-Mail: tina.ladwig@nithh.de

C. Arndt

Technische Universität Hamburg, Hamburg, Deutschland 


\section{$1 \quad$ Einleitung}

Der Beitrag gibt Einblick in das Vorgehen und ausgewählte Erkenntnisse aus dem Forschungsprojekt BRIDGING, das grundsätzlich der Frage nachgeht, wie Transfer digitaler Hochschulbildungskonzepte in Fachdisziplinen im Kontext von Hochschulverbünden verläuft.

Als Zugang wurden befristete und unbefristete formalisierte Hochschulverbünde auf Bundeslandebene im Themenfeld Digitalisierung in Studium und Lehre gewählt, in denen eine möglichst große Anzahl staatlicher Hochschulen nach den Landeshochschulgesetzen vertreten ist.

In diesem Beitrag stehen ausgehend von 34 Interviews mit 36 Verbundexpert*innen die Impulse und Motive zur gemeinsamen Gestaltung digitaler Hochschulbildung im Vordergrund sowie die Ziele, die verfolgt werden. Darüber hinaus werden die von den Interviewpartner*innen thematisierten Potenziale netzwerkübergreifender Zusammenarbeit in Deutschland näher betrachtet sowie Perspektiven für Praxis und Forschung erarbeitet. Der empirisch angelegte Beitrag versteht sich dabei unter anderem auch als Weiterführung und Ergänzung des bereits seit Langem bestehenden Austausches der Landesinitiativen im Rahmen des informellen Arbeitskreises (e-teaching.org 2016) sowie der eigenen Darstellungen der Landesinitiativen (Bremer et al. 2010).

\section{Zusammenarbeit von Hochschulen: Landeshochschulverbünde}

\subsection{Landeshochschulverbünde im Überblick}

Kooperationen im Hochschulbereich gewinnen - nicht zuletzt auch angesichts der zahlreichen Förderprogramme - weiterhin an Bedeutung. Dies ist ebenfalls im Themenfeld der digitalen Hochschulbildung und der großen Anzahl früherer und aktueller (in)formeller sowie persönlicher und institutioneller Kooperationen zu beobachten. Auf individueller Ebene finden sich motivierte Akteur*innen aus unterschiedlichen Bereichen und Fachrichtungen zu pionierähnlichen Gemeinschaften zusammen. Ebenso wird Digitalisierung in Lehre und Studium von den Hochschulleitungen mehr und mehr als Kooperationsaufgabe verstanden und im Zuge von Strategiebildung und -entwicklung entsprechend verankert (Schmid und Baeßler 2016). Darüber hinaus finden das gemeinsame Lernen und Gestalten digitaler Hochschulbildung in hochschulübergreifender Zusammenarbeit statt (Getto und Kerres 2017; Wannemacher 2016). 
Sowohl für die organisationsinterne als auch -übergreifende Zusammenarbeit hat sich der Begriff der Kooperation etabliert. In der Forschung lässt sich zwischen Kooperation, (strategischer) Allianz und (strategischem) Netzwerk unterscheiden (Zentes et al. 2005). Alle Begriffe sind auch im Hochschulbereich zu finden. Eine Studie im Auftrag des Hochschulforums Digitalisierung (HFD) zeigt dabei, dass die Mehrzahl der befragten Hochschulen an einem Verbund oder Konsortium beteiligt ist (60\%). Mehr als die Hälfte kategorisierte den Verbund/das Konsortium als landesweites Hochschulnetzwerk oder als allianz. Weitere Formen wurden als Dienstleister in Trägerschaft der Hochschulen oder des Landes (Institute oder Kompetenzzentren), zeitlich begrenzte Kooperationsprojekte und regionale oder bundesweite Hochschulnetzwerke und -allianzen beschrieben (Wannemacher et al. 2016, S. 32 f.).

Sehr weit verbreitet im Hochschulbereich ist der Begriff Verbund. Laut Duden (o. J.) wurde das Wort Bündnis im 20. Jahrhundert neu rückgebildet aus dem Verb „verbinden“ und bezeichnet unter anderem eine „bestimmte Form des Zusammenschlusses bzw. der Zusammenarbeit" (ebenda). Da nicht die durchaus interessante theoriegeleitete Analyse der Kooperationsformen im Vordergrund stand, wurde der Begriff Verbund als neutralste Bezeichnung für die Untersuchung gewählt.

Um ein möglichst konsistentes Sample in dem diversen Feld von Hochschulverbünden in Digitalisierung und Lehre zu erhalten, wurde ein weiteres Auswahlkriterium formuliert. Dieses umfasst eine aus der Selbstdarstellung erkennbare breite Beteiligung verschiedener Hochschulen und Hochschulakteur*innen an der Gestaltung eines (un)befristeten formalisierten Hochschulverbundes. Daher wurden das E-Learning Netzwerk Brandenburg (eBB) als informelles und personenbezogenes Netzwerk sowie das eTEACH-Netzwerk in Thüringen als von der Universität Weimar organisiertes Weiterbildungsprogramm für Lehrende nicht in die Interviewstudie einbezogen. Das Multimediakontor Hamburg (MMKH) als weiterer Landesverbund in Hamburg wurde als Mitglied der HOOU einbezogen.

In der ersten Projektphase (01.01.2018-31.12.2018) wurden vier der elf identifizierten Landeshochschulverbünde (Arndt et al. 2019) als Forschungsfelder ausgewählt. In der zweiten - neu beantragten - Projektphase (01.03.201930.06.2020) wurden weitere fünf einbezogen ${ }^{1}$ (siehe Abb. 1).

Um der Komplexität der Landeshochschulverbünde zu entsprechen, wurde ein qualitatives Forschungsdesign konzipiert, das die Komplexität der unterschiedlichen Ebenen möglichst differenziert abbildet. Ausgehend von einem deliberaten

\footnotetext{
${ }^{1}$ Das Projekt war ursprünglich auf ein Jahr angelegt.
} 


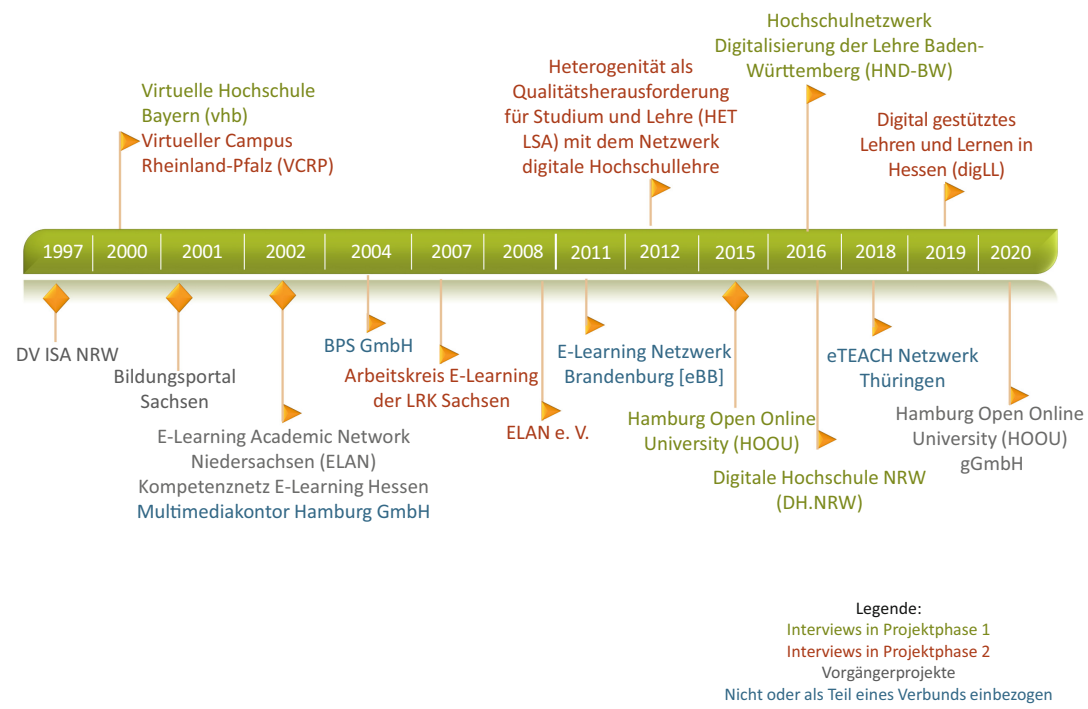

Abb. 1 Überblick BRIDGING-Sampling der aktuellen Landeshochschulverbünde im Themenfeld Digitalisierung in Studium und Lehre und ihre Historie. (Eigene Darstellung)

und damit absichtsvollen Sampling (Flick 2009, S. 122) wurden Verbundexpert*innen in den neun ausgewählten Hochschulverbünden in ihrer Rolle als strategische oder taktisch-operative Expert*innen mit entsprechend konzipierten Leitfäden befragt. ${ }^{2}$ Expert*inneninterviews werden nach Gläser und Laudel in Untersuchungen eingesetzt, ,in denen soziale Situationen oder Prozesse rekonstruiert werden sollen, um eine sozialwissenschaftliche Erklärung zu finden“ (2010, S. 13; Hervorhebung im Original). Die Leitfäden für die strategische und taktisch-operative Ebene unterscheiden sich durch einen stärkeren Fokus entweder auf die strategische Ausrichtung (Expert*innen für den Verbund - Strat_Exp) oder auf die Schnittstelle mit der eigenen Hochschule (Expert*in für die Hochschule im Verbund - Takt_Exp). Damit stand nicht die Repräsentativität der Stichprobe im Fokus der Auswahlentscheidung, sondern die Berücksichtigung diverser kontextueller Einflüsse (Flick 2009, S. 123).

Ziel der Interviews war es unter anderem, Motive, impulsgebende Akteur*innen, Ziele und Nutzen in den untersuchten Landeshochschulverbünden

${ }^{2}$ Darüber hinaus wurden 22 Interviews mit 29 Fachexpert*innen mit einem darauf angepassten Leitfaden befragt. Hier stand die Ebene der Fakultäten und Fächer im Vordergrund. 
zu identifizieren, die im Folgenden im Kontext der befragten Hochschulverbünde in den Blick genommen und um bereits bestehende Erkenntnisse ergänzt werden.

\subsection{Motive und Ziele von Landeshochschulverbünden}

Als Grundmotive für die Zusammenarbeit zwischen selbstständigen Organisationen, sei es in Allianzen oder Netzwerken, können unter anderem der Zugang zu Ressourcen, Kostenvorteile oder Leistungseffizienz gesehen werden (Zentes et al. 2005, S. 6). Kooperationen zwischen Hochschulen werden - ausgehend von dem zunehmenden Leistungsdruck im Hochschulbereich bei gleichzeitig zunehmendem Wettbewerb unter den Hochschulen - mit ähnlichen Motiven ,zur Vermeidung redundanter Strukturen und Ressourcen sowie einer Steigerung der Effizienz" (Dornseiff et al. 2016, S. 967 f.) begründet. Demnach stellt sich die Frage, inwieweit sich diese oder andere Motive in den hier vorliegenden Hochschulverbünden identifizieren lassen und welche Akteur*innen als impulsgebend beschrieben werden.

Auch in den hier vorliegenden Landeshochschulverbünden lassen sich alle der genannten Motive nachvollziehen. Hinsichtlich des Wettbewerbs lässt sich dabei beobachten, dass dieser auch auf Bundeslandebene durch einen Vergleich und ein Anschließen an die Entwicklungen in anderen Bundesländern wirksam wird. Maßgebliche Motive für die Zusammenarbeit im Themenfeld Digitalisierung sind darüber hinaus sowohl in verbreiteten Trends (Stichwort „virtuelle Hochschule“ in den 2000er-Jahren) als auch dem wahrgenommenen zunehmenden Einfluss US-amerikanischer privater Anbieter*innen zu finden. Die thematische und technische Komplexität sowie die Geschwindigkeit von Digitalisierung führten zudem zu der Überzeugung, dass diesen Herausforderungen nicht von einer einzelnen Hochschule zu begegnen ist. Hinsichtlich der Frage nach impulsgebenden Akteur*innen für die Konstituierung der Hochschulverbünde kann auf eine Untersuchung von fünf bilateralen und einer trilateralen Kooperation von Hochschulen Bezug genommen werden. Einzelne Wissenschaftler*innen in Forschung und Lehre, Akteur*innen aus der Verwaltung können demnach ebenso Impulsgeber*innen sein wie jene auf Rektorats- oder Präsidialebene (Hener et al. 2007). Ferner werden Kooperationen auch extern ausgelöst, etwa durch strategische landesund bundespolitische Vorgaben. Impulsgebende lassen sich also hochschulextern (zum Beispiel von der Landespolitik) oder hochschulintern (Hochschulen, Hochschulgremien) lokalisieren. Von den Interviewpartner*innen werden die Impulsgeber*innen in den Hochschulen, aber auch in der Landespolitik verortet. Während Hochschulvertreter*innen in fünf Verbünden und Vertretende der 
Landespolitik in einem Verbund sogar namentlich als impulsgebend benannt werden, wird in allen Verbünden auf einen Aushandlungsprozess zwischen Vertretenden der Landespolitik und der Hochschulen über die Ausgestaltung der Verbünde verwiesen. Dies zeigt, dass Impulse nicht nur landespolitischen Forderungen entstammen, aber durchaus landespolitischer Unterstützung bedürfen. Betrachtet man die historische Entwicklung der bereits Anfang der 2000erJahre gegründeten Verbünde, wird zudem die Relevanz der Unterstützer*innen aus den Hochschulen und der Landespolitik hinsichtlich der Verstetigung und der Kooperationsform deutlich. So wurden beispielsweise der Virtuelle Campus Rheinland-Pfalz (VCRP) und die Virtuelle Hochschule Bayern (vhb) als gemeinsame (wissenschaftliche) Einrichtungen im Landeshaushalt verankert, der ELAN e. V. wurde nach dem Auslaufen der sechsjährigen Landesförderung letztlich als Verein von damals acht Hochschulen weitergeführt. Das Landesprojekt Bildungsportal Sachsen wurde durch die Gründung der BPS GmbH und die Einrichtung des Arbeitskreises E-Learning der LRK für langfristigere Entwicklungen umgestaltet. Eine historische Betrachtung der Verbünde zeigt dabei, dass sich nicht nur die Organisationsformen, sondern auch die Ziele von Verbünden ändern. ${ }^{3}$

Insgesamt fokussieren die Hochschulverbünde unterschiedliche aktuelle Ziele. Diese lassen sich - auch ausgehend von ihrem Selbstverständnis - grob in zwei Bereiche differenzieren: die Förderung digitaler Lehr- und Lernangebote sowie die Förderung von Ermöglichungsstrukturen für die Hochschul(typ)en.

\subsubsection{Förderung digitaler Lehr- und Lernangebote}

In der vhb als gemeinsamer Einrichtung der Universitäten und Fachhochschulen nach Art. 1 Abs. 2 Nrn. 1 und 3 BayHSchG als „Broker“ zwischen den 31 Trägerhochschulen wird die hochschulübergreifende Entwicklung und Nutzung digitaler Lehr- und Lernangebote als Ergänzung zur Präsenzlehre angestrebt. So soll Studierenden einerseits die Gelegenheit gegeben werden, auch in digitaler Form zu studieren, ,und gleichzeitig tatsächlich eine fachliche Ergänzung und Erweiterung für die jeweiligen Fächer" (Strat_Exp) angeboten werden. Seit 2018 wurden die curricular verankerten Classic-Kurse um offen zugängliche Open-vhb-Selbstlerneinheiten für Interessierte sowie Smart-vhb-BlendedLearning-Einheiten für Lehrende zur Anreicherung der Lehrveranstaltungen ergänzt.

\footnotetext{
${ }^{3}$ Die Verbundexpert*innen weisen auf verschiedene Veränderungen und Anpassungen in den Zielen und der Struktur im Laufe der Zeit hin. So fokussierten beispielsweise der VCRP und ELAN zu Beginn ebenfalls die Er- und Bereitstellung von digitalen Lehr- und Lernmaterialien.
} 
In der HOOU als Entwicklungsprojekt von fünf Hochschulen, dem $\mathrm{UKE}^{4}$, der MMKH GmbH und der Freien und Hansestadt Hamburg wird die Entwicklung von offen lizensierten digitalen Lehr- und Lernangeboten angestrebt, die extracurriculare und experimentelle Formen des digitalen Lernens ermöglichen sollen, welche ,im Curriculum sehr schwer umsetzbar wären“ (Strat_Exp). Lernende sind dabei nicht nur Studierende, sondern alle Interessierten. Die Expert*innen verweisen bei den Zielen vor allem auf zwei Teilbereiche des entwickelten Markenkerns der HOOU: Öffnung für neue Zielgruppen und die Zivilgesellschaft sowie Openness/Open Educational Resources.

Das Landesprojekt „Digital gestütztes Lehren und Lernen in Hessen“ (digLL) mit elf Universitäten und Fachhochschulen/HAW versteht sich als ,Interessengemeinschaft“" und hat vor allem die „Erstellung und Bereitstellung digitaler Lehrund Lernmaterialien“ (Strat_Exp) zum Ziel. Die Teilprojekte an den Hochschulen verfolgen darüber hinaus jeweils spezifische Zielsetzungen mit dem Fokus auf der Unterstützung von Lehrenden. In den themenbezogenen Innovationsforen werden hochschulübergreifende Fragestellungen bearbeitet, in denen beispielsweise ,ganz klar Handlungsempfehlungen entwickelt werden sollen, die dann über die Teilprojekte wieder in die einzelnen Hochschulen zurückgespielt werden“ (Strat_Exp). digLL bewegt sich mit der gleichzeitigen strukturellen Unterstützung an den Hochschulen zwischen dieser und der nächsten Ebene - der Förderung von Ermöglichungsstrukturen.

\subsubsection{Förderung von Ermöglichungsstrukturen}

Die DH.NRW als Kooperationsgemeinschaft von 42 Hochschulen und dem Ministerium für Kultur und Wissenschaft strebt in ihren Handlungsfeldern Studium \& Lehre, Forschung sowie Infrastruktur \& Management auf die hochschulübergreifende Aushandlung, Gestaltung und Verfügbarmachung vorhandener und zukünftiger Entwicklungen und Lösungen an. Der Verbund zielt damit nicht unmittelbar auf die Entwicklung von digitalen Lehr- und Lernangeboten, sondern darauf, vorhandene Entwicklungen und Lösungen , in die Fläche zu bringen und sich darüber hinaus auch Gedanken zu machen, was vielleicht ganz neue Strukturen, Services und Angebote für den Hochschulstandort NRW bedeuten können“ (Strat_Exp). Dabei wird betont, dass nicht nur gemeinsame Ziele identifiziert,

\footnotetext{
${ }^{4}$ Zum Zeitpunkt der Interviews war die Universität Hamburg (UHH) Teil des Landesprojektes HOOU. Die HOOU wurde 2019 verstetigt. Seit April 2019 ist die UHH nicht mehr Teil der HOOU-Gremien und hat auch kein Teilprojekt.
} 
sondern auch Umsetzungsansätze durch ,gemeinsame Projekte [...], die schlagkräftig sind und die die Hochschulen alle gemeinsam voranbringen“"(Strat_Exp), entwickelt werden sollen.

Das Ziel des HND-BW als Netzwerk von und für 49 Hochschulen kann mit der hochschultypenspezifischen und -übergreifenden Bearbeitung von Themen und (technischen) Rahmenbedingungen zusammengefasst werden. Die ansonsten oft separat agierenden Hochschultypen sprechen dafür ,in einer organisierten Weise miteinander über das Digitalisierungsthema“ (Strat_Exp). Neben gemeinsamen Entwicklungen vor allem im Kontext der Themengruppen zielt das HND-BW darauf, Impulse und Empfehlungen sowohl an Hochschulleitungen als auch an das Wissenschaftsministerium zu geben. ${ }^{5}$

Das Ziel des VCRP als wissenschaftliche Einrichtung und Netzwerkeinrichtung der zwölf Universitäten und Fachhochschulen/HAW nach § 93 HochSchG wird damit beschrieben, „Unterstützung, Vernetzung, aber auch Innovationsentwicklung durch Forschung und Entwicklung“ (Strat_Exp) zu ermöglichen. Im Rahmen der drei Handlungsfelder Service \& Support, Vernetzung sowie Innovation \& Forschung geht es dabei ,nicht unbedingt primär [darum,] die digitale Bildung voranzubringen, sondern [...] zu ermöglichen, dass die Unis und Hochschulen das können“ (Takt_Exp).

Im ELAN e. V. mit zehn Mitgliedshochschulen wird das Ziel in der Unterstützung der Hochschulen in ihrem Bedarf und ihren Zielsetzungen und der Kommunikation mit der Landespolitik verdeutlicht, um damit „die Rahmenbedingungen für das zu verbessern, was die einzelnen Hochschulen machen wollen in digitaler Lehre“ (Strat_Exp). Dies wird im Zusammenhang mit der Rechtsform als Verein auch als Konzentration auf „Politik und Nachhaltigkeit“" (Takt_Exp) zusammengefasst. Die Arbeit orientiert sich an fünf Kompetenzbereichen, die unter anderem aus den Förderphasen vor der Vereinsgründung übernommen wurden: Lernmanagementsysteme, Rechtsfragen, E-Assessment, Audiovisuelle Medien und Mediendidaktik.

Im Verbundprojekt HET LSA mit sieben Hochschulen und dem HoF werden die strategischen Ziele klar damit beschrieben ,Studienabbruch [zu] senken [und] sich attraktiver [zu] machen für neue Zielgruppen“ (Strat_Exp). Die Teilprojekte an den Hochschulen verfolgen dabei eigene Teilthemen und -ziele. Die Transferstelle dient unter anderem explizit der Förderung funktions- und themenbezogener Zusammenarbeit der Hochschulen. In diesem Zusammenhang initiierte

\footnotetext{
${ }^{5}$ Nach Auslaufen der Landesförderung übernahmen die Universitäten die Weiterfinanzierung der Geschäftsstelle für weitere drei Jahre. Am HND-BW sind nur noch die Universitäten beteiligt (vgl.: www.hnd-bw.de/2019/10/14/rueckblick-hnd-bw-jahreskonferenz-2019universitaet-stuttgart/).
} 
das Teilprojekt an der Universität Halle die AG E-Learning, die 2017 in „Netzwerk digitale Hochschullehre“ umbenannt wurde. Als „kleines Netzwerk im Netzwerk“ (Takt_Exp) hat es zum Ziel, Informationen bereitzustellen, Qualifizierungsangebote für Lehrende umzusetzen sowie strukturelle Rahmenbedingungen für Digitalisierung zu ermöglichen.

Das Bildungsportal Sachsen kann als Landesinitiative von drei Hochschulverbünden gesehen werden: dem Arbeitskreis E-Learning, der BPS GmbH und dem Hochschuldidaktischen Zentrum Sachsen (HDS). Der hier untersuchte 2007 als Kommission der Landesrektorenkonferenz und wissenschaftliches Gremium gegründete Arbeitskreis E-Learning mit Mitgliedern und Gästen aus 15 Hochschulen zielt darauf, „E-Learning-Aktivitäten der sächsischen Hochschulen zu bündeln und zu koordinieren“ (Takt_Exp). Ähnlich wie im ELAN e. V. werden die operativen Ziele in der Regel alle zwei Jahre auch ausgehend davon, ,welche Ziele die jeweilige Hochschule sozusagen gerade erreichen möchte oder wie der Diskurs dazu geführt wird" (Strat_Exp), im Arbeitskreis diskutiert. In diesem Zusammenhang ist auch die Koordination der Landesförderung zu sehen, bei der der Arbeitskreis der LRK zuarbeitet. Dabei entwickelt er unter anderem Konzepte, konzipiert Ausschreibungen für Hochschulprojekte und arbeitet in der Umsetzung eng mit dem HDS und der BPS GmbH zusammen, welche als Unternehmen der Dienstleister für die Hochschulen das klare Ziel verfolgt, ,eine technologische Basis zu schaffen, die für alle nutzbar ist" (Takt_Exp).

Übergreifend wird in fast allen Hochschulverbünden das Ziel deutlich, dass „das Not-invented-here-Syndrom ein bisschen aufgefangen wird, dass man sich vernetzt" (Takt_Exp). Neben den unterschiedlichen Zielsetzungen spiegeln sich übergreifend dieselben Ziele wider, die Hener et al. (2007) in ihrer Studie zu Hochschulkooperationen als Synergie- und Breitenwirkung sowie politischstrukturelle Ziele zusammengefasst haben. Letztere fokussieren die Ebene der Landespolitik, der Hochschulleitung, der Fakultäten/Fachbereiche oder der Individuen an den Hochschulen. In BRIDGING werden diese Ebenen unter der Fragestellung der Transferstrategien oder der jeweiligen Zielsysteme aktuell detaillierter analysiert.

Grundsätzlich besteht ein Zusammenhang zwischen den Zielsetzungen und dem Nutzen, der der Zusammenarbeit im Verbund zugeschrieben wird. Wahrgenommener Nutzen führt einerseits zu der Konstitution und den Zielsetzungen von Hochschulverbünden, kann aber auch über diese hinaus von Hochschulvertreter*innen wahrgenommen werden. Motive für die Konstituierung und Ziele von Verbünden und der in der Zusammenarbeit festgestellte Nutzen können identisch sein, sind es oft auch, müssen es aber nicht. Übergreifend lassen sich 
in den Interviews erhoffte und eingetretene Nutzeffekte in drei Oberkategorien differenzieren:

- Bündelung der Ressourcen

- Motor und Austauschort

- Verbesserung der Rahmenbedingungen

Die Bündelung der Ressourcen umfasst Aussagen, in denen vor allem technische, finanzielle und personell-fachliche Ressourcen erwähnt werden, die einzelnen Hochschulen in dem Umfang nicht zur Verfügung stehen würden. Unter Motor und Austauschort wurden solche Aussagen gefasst, in denen thematischinhaltliche und persönliche Verbesserungen wie Freiräume für neue Ideen oder das Lernen voneinander angesprochen werden. Die Verbesserung der Rahmenbedingungen bezieht sich auf rechtlich-strukturelle bundes- oder landesweite Verbesserungen, die unabhängig von spezifischen Zielen oder Bedarf einer Hochschule alle Hochschulen betreffen, wie beispielsweise rechtliche Regelungen. Auffällig ist, dass weder Ziele noch Nutzen rein ökonomisch argumentiert werden, wenngleich oftmals auf die (finanziellen) Ressourcen eingegangen wird. Diese Tatsache gewinnt wiederum an Relevanz angesichts der Erkenntnisse von Hener et al. (2007, S. 8), dass bereits die Vermutung einer finanziellen Einsparung, ohne eigenen Nutzen als Hauptziel, eine erfolgreiche Zusammenarbeit gefährdet.

\section{$3 \quad$ Leitideen und Umsetzungsansätze der Hochschulverbünde}

Es stellt sich neben den Motiven, Zielen und dem Nutzen zudem die Frage nach den Konzepten, die die Hochschulverbünde für die digitale Hochschulbildung entwickeln und verfolgen. Digitale Hochschulbildungskonzepte werden hier in Anlehnung an Pasternack et al. (2018) als hochschulsystembezogene Leitideen und Umsetzungsansätze für die Gestaltung akademischer Bildung(seinrichtungen) im Kontext der digitalen Transformation verstanden. Verbundübergreifend können vier Leitideen identifiziert werden, die mit unterschiedlichen Ausprägungen und Umsetzungsansätzen in Form von Formaten, Prozessen und Produkten gestaltet werden. Die Leitideen sind als analytisch getrennt zu betrachten. In der Praxis der Verbünde stehen sie in vielfältigen Beziehungen zueinander; sie weisen Verbindungen untereinander und zu den beschriebenen Zielen auf. 


\section{Teilen}

Unter der Leitidee des Teilens sind verschiedene Aspekte zusammengefasst, in denen es um die Entwicklung und Bereitstellung von Bildungsmaterialien oder Lehr- und Lernangeboten geht, die mit anderen geteilt werden. Die anderen sind dabei schwerpunktmäßig andere Hochschulen und Lehrende (beispielsweise in der vhb). Je nach Verbund geht es jedoch auch darum, Strukturen und Services von Hochschulen zu identifizieren und diese mit anderen Hochschulen zu teilen. Eine weitere Facette des Teilens ist im Zusammenhang mit den Verbünden als Motor und Austauschort zu sehen, wodurch Erfahrungen und Ideen zwischen Lehrenden, Beratenden und Hochschulen geteilt werden. Umsetzungsansätze sind dabei neben strukturellen Einheiten, die das Teilen von Expertise und Erfahrungen ermöglichen, wie in den Kompetenzzirkeln im HET LSA das Teilen einer gemeinsamen technischen Infrastruktur wie OPAL im Bildungsportal Sachsen oder das Teilen von Lehr- und Lernmaterialien über OER-Plattformen wie im HND-BW.

2. Öffnung und Offenheit

Unter der Leitidee der Öffnung und Offenheit wurden vor allem Aspekte zusammengefasst, die mit einer Öffnung von akademischen Lehr- und Lernangeboten einhergehen. Die Zielgruppen umfassen dabei sowohl sogenannte nicht traditionelle Studierende, Studierende anderer Hochschulen als auch Lehrende sowie Personen, die weder an einer Hochschule studieren noch arbeiten. Oft, aber nicht nur ist dies ebenfalls verbunden mit OER oder Open Source. Offenheit umfasst auch ein Bewusstsein von Hochschulen und Hochschulangehörigen, extern vorhandene Expertise und Angebote zu sehen und aufzunehmen. Umsetzungsansätze sind dabei unter anderem die konsequente Verfügbarmachung von OER-Lernangeboten in der HOOU für alle Interessierten, aber auch bestehender und eigener Open-Source-Software-Entwicklungen im VCRP.

3. Kollaboration

Bei der Leitidee der Kollaboration geht es - neben dem konstitutiven Merkmal von Verbünden - einerseits um die Zusammenarbeit der Hochschulen bei der Entwicklung von Lehr- und Lehrangeboten sowie Services und Strukturen, andererseits um die Zusammenarbeit von Lehrenden und/oder Lernenden. Besondere Erwähnung findet hierbei die Schaffung von Communities of Practice (Wenger 2010). Dies wird beispielsweise in kollaborativen Projekten zwischen mindestens zwei Hochschulen sowohl in der DH.NRW als auch dem Bildungsportal Sachsen und der vhb umgesetzt. Im digLL und dem HNDBW wird Kollaboration von den Innovationsforen oder den Themengruppen umgesetzt. Im Wesentlichen zielt diese Leitidee auf die Zusammenarbeit in 
der Entwicklung ab, während es bei der Leitidee des Teilens stärker um die übergreifende Zugänglich- oder Nutzbarmachung von Entwicklungen und Angeboten geht.

4. Adressat*innenorientierung

Die Leitidee der Adressat*innenorientierung ${ }^{6}$ bedeutet einerseits eine Perspektivübernahme des potenziellen Gegenübers bei der Entwicklung von Lernangeboten und andererseits die Selbstbestimmung eines Lernprozesses. Ausgehend von den technischen Umgebungen in den Verbünden werden auch die Perspektiven von Nutzer*innen - sowohl Lehrende als auch Lernende - als zunehmend leitend für (Weiter-)Entwicklungen thematisiert. Ein Umsetzungsansatz lässt sich im HET LSA, der sich explizit mit Heterogenität beschäftigt, in der Entwicklung eines hochschulübergreifenden BlendedLearning-Weiterbildungsangebotes für Lehrende zur heterogenitätssensiblen Seminargestaltung finden.

Trotz zahlreicher strategischer und struktureller Unterschiede fokussieren die Hochschulverbünde ähnliche Umsetzungsansätze und Themenfelder und sind mit ähnlichen Problemstellungen konfrontiert, die ganz im Sinne der Leitideen auch miteinander geteilt, füreinander geöffnet und weiterentwickelt werden können. So werden (digitale) Qualifizierungsangebote für Lehrende, digitale akademische Lernangebote für Studierende und Lernende oder Austauschformate für Lehrende und Berater*innen und vor allem Plattformen oder OER-Repositorien zum Teilen von Lehr- und Lernmaterialien angesprochen. Problemstellungen sind dabei unter anderem das Urheberrecht, die Anrechnung digitaler Lehre auf das Lehrdeputat, die Schaffung von Anreizsystemen sowie die Kommunikation im Verbund über die Verbundgremien hinaus. Während diese in BRIDGING unter dem Aspekt von Faktoren, die Transferprozesse im Kontext von Hochschulverbünden fördern oder behindern, aktuell untersucht werden, soll im Folgenden auf thematische Schnittstellen, ähnliche Problemstellungen und sich daraus ergebende Synergiepotenziale eingegangen werden.

\footnotetext{
${ }^{6}$ Der Begriff ist teilweise fachdisziplinär geprägt und wird unter anderem in der Linguistik verwendet wie beispielsweise von Becker-Mrotzek et al. (2014).
} 


\section{$4 \quad$ Schnittstellen und Synergien zwischen Landeshochschulverbünden}

Um den Hinweisen aus den Interviews zu Synergien netzwerkübergreifender Zusammenarbeit nachzugehen, wurde 2019 eine (nicht repräsentative) Umfrage gestartet, die sich vor allem an Hochschulen und Akteur*innen richtet, die Teil eines hochschulübergreifenden Netzwerkes im Kontext digitaler Hochschulbildung $\operatorname{sind}^{7}$ (Ladwig et al. 2020). Ausgehend von ähnlichen Themenfeldern und Problemstellungen soll der finale Stand dargestellt werden, in welchen Netzwerkakteur*innen den Austausch und die Zusammenarbeit ihrer hochschulübergreifenden Netzwerke mit anderen hochschulübergreifenden Netzwerken stärken würden. Es geht damit um die Frage, in welchen Themenfeldern Netzwerke, nicht Einzelakteur*innen, zusammenarbeiten sollten (Abb. 2).

Die Themenfelder Austausch von Inhalten von Lehrenden und Strategieentwicklung im Themenfeld Digitalisierung und Lehre konnten ihre führende Stellung beibehalten. Die dritt- und viertgenannten Punkte Anrechenbarkeit auf das Lehrdeputat und Erstellung digitaler Lehr- und Lernkonzepte haben sich in der finalen Auswertung umgekehrt. E-Prüfungen und rechtliche Themenfelder werden ebenfalls weiterhin mit am meisten genannt. In der Detailauswertung der strategischen Netzwerkarbeit sind weiterhin die Themenfelder Infrastrukturelle Zusammenführung von Lehre und Forschung (FDM) sowie die Zusammenarbeit mit mittelgebenden Ministerien und Behörden und die Nachhaltigkeit von (Förder-)Projekten im Netzwerk meistgenannt. Ebenso haben sich die meistgenannten Mehrwerte und Nutzen, den die Befragten einer netzwerkübergreifenden Zusammenarbeit zuschreiben, nicht verändert (siehe Abb. 3).

Die zugeschriebenen Nutzeffekte hinsichtlich einer netzwerkübergreifenden Zusammenarbeit bieten sich für einen Vergleich mit den Daten aus den Expert*inneninterviews und denen der Umfrageteilnehmenden über den Nutzen ihrer eigenen hochschulübergreifenden Netzwerke an.

Im Vergleich fällt auf, dass diese ihren hochschulübergreifenden Netzwerken Effekte bezüglich der Erweiterung ihrer (persönlichen) Kompetenzen zuschreiben. Dies entspricht dem Nutzen der Hochschulverbünde als Motor und Austauschort. Auch der Ausgleich von Nachteilen (aufgrund von Größe, Ressourcen etc.) wird als Vorteil der hochschulübergreifenden Netzwerke gesehen, was in den Interviews unter anderem unter der Verbesserung der Rahmenbedingungen in Form einer gleichberechtigten Teilhabe an Förderprogrammen thematisiert wird.

\footnotetext{
${ }^{7}$ Über die hier bereits genannten Landeshochschulverbünde wurden auch Landeshochschuldidaktikzentren und Hochschulverbünde wie die Virtuelle Fachhochschule angesprochen.
} 


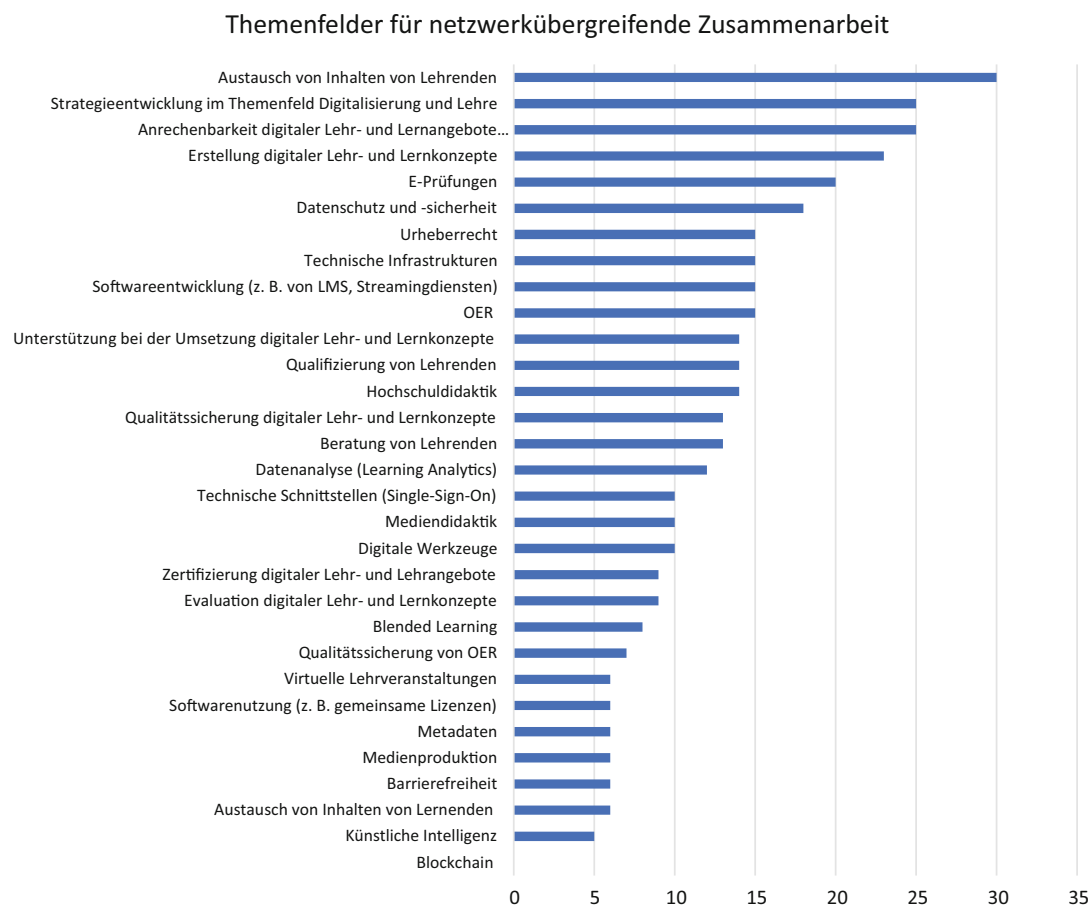

Abb. 2 Themenfelder für netzwerkübergreifende Zusammenarbeit. (Eigene Darstellung)

Was wäre jedoch der Nutzen einer Vernetzung von Netzwerken? Dieser liegt angesichts der vorliegenden Daten (Abb. 3) insbesondere in der Erzielung größerer Wirkung im Vergleich zu den eigenen hochschulübergreifenden Netzwerken.

Insbesondere ausgehend von den hier fokussierten Landeshochschulverbünden zeigt dies, dass netzwerkübergreifende Zusammenarbeit nicht dasselbe leisten kann oder ggf. zu leisten versuchen sollte, wie es in den einzelnen Hochschulverbünden ermöglicht wird. Bestimmte Mehrwerte können jedoch skaliert 


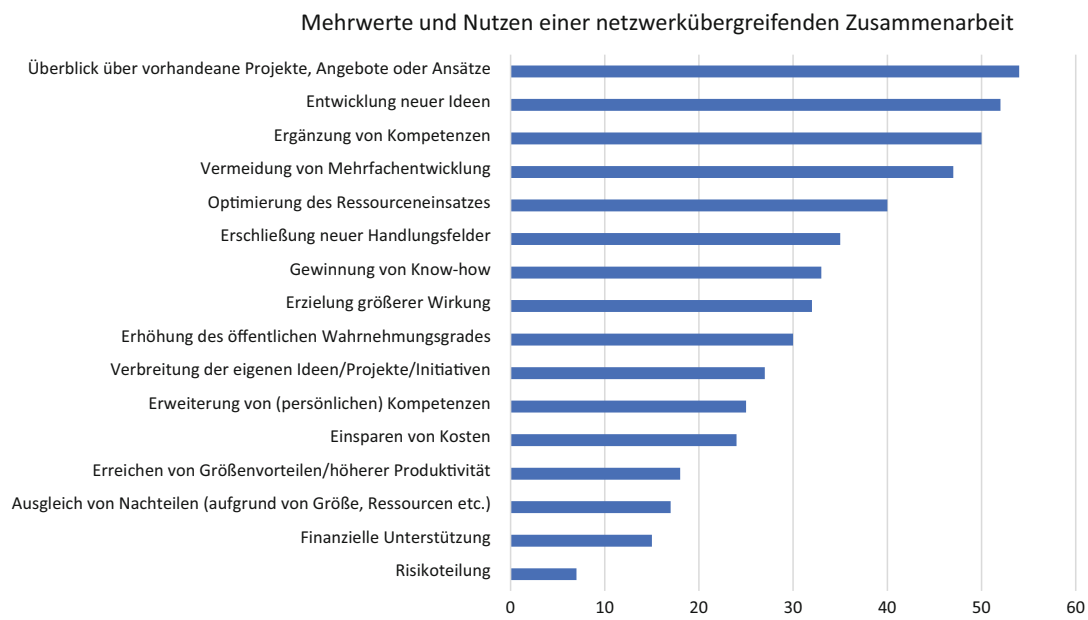

Abb. 3 Mehrwerte und Nutzen einer netzwerkübergreifenden Zusammenarbeit. (Eigene Darstellung)

oder besonders fokussiert werden. ${ }^{8}$ Dabei ist ausgehend von den Erkenntnissen sowohl aus den Interviews als auch der Umfrage zu berücksichtigen, dass die hier vorgestellten Landeshochschulverbünde nicht die einzigen Initiativen im Hochschulbereich sind, die Digitalisierung in Studium und Lehre gestalten. Insbesondere die Hochschuldidaktikzentren und der Dachverband Deutsche Gesellschaft für Hochschuldidaktik (dghd) oder „Lehre hoch n“ werden von den Interviewpartner*innen und den Umfrageteilnehmenden als wichtige netzwerkübergreifende Initiativen benannt, die sowohl auf Bundesland- als auch Bundesebene in die Diskussion einbezogen werden könnten. ${ }^{9}$ Etwaige Nutzenerwägungen sollten daher auch um Überlegungen zu den Zielen, Zielgruppen, Ebenen und entsprechenden Beteiligungsformaten ergänzt werden.

\footnotetext{
${ }^{8}$ In diesem Zusammenhang organisierte BRIDGING am 05.03.2020 einen Workshop aller Landeshochschulverbünde, um die Möglichkeiten einer netzwerkübergreifenden Zusammenarbeit zu konkretisieren. Die Ergebnisse wurden dokumentiert und das weitere mögliche Vorgehen spezifiziert.

${ }^{9}$ Nicht in allen Bundesländern existieren Hochschuldidaktikzentren.
} 


\section{$5 \quad$ Ausblick}

Trotz der zahlreichen Informationsplattformen besteht weiterhin ein großes Bedürfnis der Akteur*innen nach mehr Transparenz und Zugänglichkeit wie auch einem geprüften strukturierten, kontext- und zielgruppenspezifischen, aber auch technisch nachhaltigen Über- und Einblick in vorhandene Projekte und Angebote. Dies gilt es entsprechend in zukünftigen weiteren Forschungs- und Entwicklungsansätzen - neben der Entwicklung von weiteren innovativen Ansätzen und Angeboten - zu berücksichtigen.

Für den Auf- und Ausbau, die Weiterführung und Gestaltung von Hochschulverbünden und netzwerkübergreifender Zusammenarbeit bedarf es neben einem Überblick zudem weiterer Forschungsbemühungen. Interessanterweise sind trotz der hohen praktischen Relevanz des Phänomens kooperativer Initiativen zwischen Hochschulen im Themenfeld Digitalisierung in Studium und Lehre die Forschungsbemühungen bisher überschaubar. Mögliche Erklärungen hierfür könnten einerseits darin liegen, dass sie initiiert wurden, um praktische und vor allem infrastrukturelle Probleme zu bearbeiten, und demnach übergreifend kaum einen Forschungsgegenstand darstellten. In Kombination mit zunehmendem Interesse an Fragen der Governance im Bildungsbereich sowie informationstechnologischen Möglichkeiten erhöht sich die Aufmerksamkeit für die Bedeutung von Hochschulkooperationen im Themenfeld von Digitalisierung in Studium und Lehre und vor allem die Synergiepotenziale zunehmend.

Bereits einzelne Hochschulverbünde unterschiedlicher Ausrichtung stellen ein umfangreiches Forschungsfeld dar, in dem beispielsweise Fragen nach der Governance hochschul- und netzwerkübergreifender Zusammenarbeit untersucht werden, wobei Größe, Struktur und Zeit entsprechende Relevanz zukommen. Um auch größere Hochschulverbünde nachhaltig zu gestalten und die Weiterführung vieler entwickelter Formate, Prozesse und Produkte zu gewährleisten, könnten empirische Erkenntnisse kleinerer Verbünde genutzt werden, die auf die Relevanz der frühzeitigen Thematisierung von Verbundstrukturen hinweisen (Maschwitz et al. 2019). Ergänzt werden kann dies durch Erkenntnisse zur partizipativen Findung und Gestaltung einer Organisations- und Rechtsform (Wagner et al. 2020) sowie zu potenziellen Konfliktlinien (Winde et al. 2019). Dabei können diesen Forschungsstand aufnehmende und vor allem begleitende qualitative und quantitative Untersuchungen einen Beitrag für die weitere Gestaltung der Zusammenarbeit für bestehende und zukünftige Initiativen leisten. Entwicklungen im Themenfeld von Digitalisierung in Studium und Lehre stellen oftmals die traditionelle Trennung von Wissenschaft und Praxis aufgrund ihrer praxisorientierten Ausrichtung infrage. In diesem Zusammenhang können Verbünde von Erkenntnissen 
bezüglich partizipativer Transferstrategien oder Design-Based-Research-Ansätzen (Gräsel 2010; Reinmann 2005), die einerseits die Relevanz der Zielsysteme herausstellen und andererseits Forschung und Praxis integrativ denken, profitieren und diese wiederum bereichern. Während BRIDGING bezüglich der Transferstrategien weitere Erkenntnisse generieren wird, ergibt sich hinsichtlich der von den Netzwerk- und Verbundakteur*innen erwünschten und sinnvoll erachteten synergetischen Gestaltung einer netzwerkübergreifenden Zusammenarbeit vor allem die Herausforderung, eine zentral-dezentral thematisch strukturierte Arbeitsorganisation zu finden, die vereinbar ist mit den derzeitigen politisch-rechtlichen Rahmenbedingungen für eine bundeslandübergreifende Zusammenarbeit in der digitalen Hochschulbildung. Wie dies und gegebenenfalls eine Anbindung an internationale Netzwerke angesichts der bereits zahlreichen bestehenden Netzwerke in Deutschland gelingen kann, könnte Gegenstand eines konzeptionellen Pilotprojekts sein.

\section{Literatur}

Arndt, C., Figura, L., \& Ladwig, T. (2019). Überblick über Landeshochschulinitiativen zur Digitalisierung. Hamburg: TUHH. https://doi.org/10.15480/882.2387.4

Bayerisches Hochschulgesetz (BayHSchG) vom 23. Mai 2006 (GVB1. S. 245, BayRS 22101-1-WK), das zuletzt durch $\S 1$ des Gesetzes vom 24. Juli 2020 (GVB1. S. 382) geändert worden ist Duden (Hrsg.). (o. J.). Verbund - Rechtschreibung, Bedeutung, Definition, Herkunft. https://www.duden.de/rechtschreibung/Verbund/. Zugegriffen: 16. Sept. 2020.

Becker-Mrotzek, M., Grabowski, J., Jost, J., Knopp, M., \& Linnemann, M. (2014). Adressatenorientierung und Kohärenzherstellung im Text. Zum Zusammenhang kognitiver und sprachlicher realisierter Teilkompetenzen von Schreibkompetenz. Didaktik Deutsch, 20(37), 21-43.

Bremer, C., Göcks, M., Rühl, P., \& Stratmann, J. (Hrsg.). (2010). Landesinitiativen für ELearning an deutschen Hochschulen. Münster: Waxmann.

Dornseiff, K., Groening, Y., \& Kassanke, S. (2016). Gelebte Kooperationsformen zwischen Hochschulen - Möglichkeiten der Ausgestaltung kritischer Erfolgsfaktoren anhand von Praxisbeispielen. In K. Dornseiff, H. C. Mayr \& M. Pinzger (Hrsg.), INFORMATIK 2016 (S. 967-979). Bonn: Gesellschaft für Informatik e. V. https://dl.gi.de/handle/20.500.121 16/969. Zugegriffen: 16. Sept. 2020.

e-teaching.org. (2016). Konzeptpapier für länderübergreifende Kooperationen. eteaching.org. https://www.e-teaching.org/projekt/politik/laenderzentren/konzeptpapierfuer-laenderuebergreifende-kooperationen/index_html/. Zugegriffen: 16. Sept. 2020.

Flick, U. (2009). An introduction to qualitative research. Los Angeles: Sage Publications.

Getto, B., \& Kerres, M. (2017). Akteurinnen/Akteure der Digitalisierung im Hochschulsystem: Modernisierung oder Profilierung? Zeitschrift für Hochschulentwicklung, 12(1), 123-142. 
Gläser, J., \& Laudel, G. (2010). Experteninterviews und qualitative Inhaltsanalyse als Instrumente rekonstruierender Untersuchungen. Wiesbaden: Springer VS.

Gräsel, C. (2010). Stichwort: Transfer und Transferforschung im Bildungsbereich. Zeitschrift für Erziehungswissenschaft, 13(1), 7-20. https://doi.org/10.1007/s11618-010-0109-8.

Hener, Y., Eckardt, P., \& Brandenburg, U. (2007). Kooperationen zwischen deutschen Hochschulen (Arbeitspapier, 85). Gütersloh: CHE. https://www.che.de/projekt/strategische-pot entiale-bei-kooperationen/. Zugegriffen: 16. Sept. 2020.

Hochschulgesetz (HochSchG) vom 19. November 2010 (GVB1. S. 463, 464), das zuletzt durch Artikel 4 des Gesetzes vom 18. Juni 2019 (GVB1. S. 101) geändert worden ist.

Ladwig, T., Arndt, C., \& Djabarian, Y. (2020). Vernetzung von Netzwerken in digitalen Zeiten: Ein Diskussionspapier. Diskussionspapier, 8. Berlin: Hochschulforum Digitalisierung. https://doi.org/10.5281/zenodo.4282343.

Maschwitz, A., Speck, K., Brinkmann, K., Johannsen, M., \& von Fleischbein, A. (2019). Nachhaltigkeit von Verbundprojekten-Ergebnisse einer Mixed-Methods-Studie. Thematischer Bericht der wissenschaftlichen Begleitung des Bund-Länder-Wettbewerbs „Aufstieg durch Bildung: offene Hochschulen “. https://nbn-resolving.org/urn:nbn:de:0111-pedocs167726. Zugegriffen: 16. Sept. 2020.

Pasternack, P., Hechler, D., \& Henke, J. (2018). Die Ideen der Universität: Hochschulkonzepte und hochschulrelevante Wissenschaftskonzepte. Bielefeld: UniversitätsVerlagWebler.

Reinmann, G. (2005). Innovation ohne Forschung? Ein Plädoyer für den Design-Based Research-Ansatz in der Lehr-Lernforschung. Unterrichtswissenschaft, 33(1), 52-69.

Schmid, U., \& Baeßler, B. (2016). Strategieoptionen für Hochschulen im digitalen Zeitalter (Arbeitspapier, 29). Berlin: Hochschulforum Digitalisierung. https://doi.org/10.5281/zen odo.4282185.

Wagner, N., Nieveler, S., \& Kessler, M. S. (2020). Clever konfigurieren: Hochschulkooperationen die geeignete Form geben. Essen: Stifterverband für die Deutsche Wissenschaft e. V. https://www.stifterverband.org/medien/clever-konfigurieren. Zugegriffen: 16. Sept. 2020.

Wannemacher, K. (2016). Digitale Modelle internationaler Hochschulkooperation in der Lehre (Arbeitspapier, 22). Berlin: Hochschulforum Digitalisierung. https://doi.org/10. 5281/zenodo.4282206.

Wannemacher, K., Jungermann, I., Osterfeld, S., Scholz, J., \& von Villiez, A. (2016). Organisation digitaler Lehre in den deutschen Hochschulen (Arbeitspapier, 21). Berlin: Hochschulforum Digitalisierung. https://doi.org/10.5281/zenodo.4282224.

Wenger, E. (2010). Communities of practice and social learning systems: The career of a concept. In C. Blackmore (Hrsg.), Social learning systems and communities of practice (S. 179-198). London: Springer London.

Winde, M., Wagner, N., Nieveler, S., Dauchert, A., \& Kleimann, B. (2019). Kooperationsgovernance. Diskussionspapier, 1. Essen: Stifterverband für die Deutsche Wissenschaft e. V. https://www.stifterverband.org/medien/kooperationsgovernance-01. Zugegriffen: 16. Sept. 2020.

Zentes, J., Swoboda, B., \& Morschett, D. (2005). Kooperationen, Allianzen und Netzwerke - Entwicklung der Forschung und Kurzabriss. In J. Zentes, B. Swoboda, \& D. Morschett (Hrsg.), Kooperationen, Allianzen und Netzwerke: Grundlagen-Ansätze - Perspektiven (S. 3-32). Wiesbaden: Gabler Verlag. 
Open Access Dieses Kapitel wird unter der Creative Commons Namensnennung 4.0 International Lizenz (http://creativecommons.org/licenses/by/4.0/deed.de) veröffentlicht, welche die Nutzung, Vervielfältigung, Bearbeitung, Verbreitung und Wiedergabe in jeglichem Medium und Format erlaubt, sofern Sie den/die ursprünglichen Autor(en) und die Quelle ordnungsgemäß nennen, einen Link zur Creative Commons Lizenz beifügen und angeben, ob Änderungen vorgenommen wurden.

Die in diesem Kapitel enthaltenen Bilder und sonstiges Drittmaterial unterliegen ebenfalls der genannten Creative Commons Lizenz, sofern sich aus der Abbildungslegende nichts anderes ergibt. Sofern das betreffende Material nicht unter der genannten Creative Commons Lizenz steht und die betreffende Handlung nicht nach gesetzlichen Vorschriften erlaubt ist, ist für die oben aufgeführten Weiterverwendungen des Materials die Einwilligung des jeweiligen Rechteinhabers einzuholen. 